\title{
Accurate Single-Valued Double Many-Body Expansion Potential Energy Surface for Ground-State $\mathrm{HN}_{2}$
}

\author{
L. A. Poveda and A. J. C. Varandas* \\ Departamento de Quimica, Universidade de Coimbra, 3004-535 Coimbra, Portugal
}

Received: May 5, 2003; In Final Form: July 11, 2003

\begin{abstract}
A single-valued double many-body expansion potential energy surface is reported for ground-state $\mathrm{HN}_{2}$ by fitting accurate ab initio energies that have been suitably corrected by scaling for the $\mathrm{H}-\mathrm{N}_{2}$ and $\mathrm{N}-\mathrm{NH}$ regions. The energies of $\sim 900$ geometries have been calculated at the multireference configuration interaction level, using aug-cc-pVQZ basis sets of Dunning and the full valence complete active space wave function as reference. The topographical features of the novel global potential energy surface are examined in detail.
\end{abstract}

\section{Introduction}

Several experimental and theoretical studies have attempted to elucidate the possible role of the $\mathrm{HN}_{2}$ species as a critical step in various reactions. Although this molecule has never been observed experimentally, it has been postulated to be an important intermediary in thermal $\mathrm{De}-\mathrm{NO}_{x}$ processes, ${ }^{1,2}$ with indirect evidence that suggests its existence through the three possible product channels proposed for the $\mathrm{NH}_{2}+\mathrm{NO}$ reaction:

$$
\mathrm{NH}_{2}+\mathrm{NO} \rightarrow\left\{\begin{array}{l}
\mathrm{HN}_{2}+\mathrm{OH} \\
\mathrm{N}_{2}+\mathrm{H}+\mathrm{OH} \\
\mathrm{N}_{2}+\mathrm{H}_{2} \mathrm{O}
\end{array}\right.
$$

The experiments that are focused to determine the rate coefficient and the major pathway of reaction 1 are far from consistent: although the experimental studies vary widely, in regard to the branching ratio for $\mathrm{OH}$ production, none of the experiments observed $\mathrm{H}$ atoms. ${ }^{3}$ This result, along with the assumed product channels, argues for the existence of the $\mathrm{HN}_{2}$ species. However, a controversy persists from the kinetics modeling studies. ${ }^{2,4}$ In fact, although an estimate of $10^{-4} \mathrm{~s}$ for the lifetime produces a reasonable match to experimental observations, the theoretical studies of $\mathrm{HN}_{2}$ unimolecular decay predict a much shorter value, of $10^{-8}-10^{-9} \mathrm{~s}$, for the ground vibrational state..$^{5-7}$

Many ab initio studies of the reaction

$$
\mathrm{H}+\mathrm{N}_{2} \rightarrow \mathrm{HN}_{2}
$$

have previously been made to characterize the geometry and energetics of the reactants, transition state, and products. ${ }^{3}$ All previous calculations concluded that the title system is quasibound (the global minimun is located above the $\mathrm{H}+\mathrm{N}_{2}$ asymptote) with a barrier height to dissociation of $\sim 11 \mathrm{kcal} /$ mol. From such studies, a global potential energy surface was reported by Walch $^{5,8,9}$ and Koizumi et al. ${ }^{6}$ for the $\mathrm{H}+\mathrm{N}_{2}$ channel. On the basis of a spline fit of the $a b$ initio points calculated using the completed active space self-consistent field/ externally contracted configuration interaction (CASSCF/CCI) method and two different basis sets, Walch and co-workers

* Author to whom correspondence should be addressed. E-mail address: varandas@qtvs1.qui.uc.pt.

10.1021/jp030571o CCC: \$25.00 predicted, depending on the basis set used, a barrier height of 10.2 or $11.3 \mathrm{kcal} / \mathrm{mol}$ and a global minimum of 5.6 or $3.9 \mathrm{kcal} /$ mol above the $\mathrm{H}+\mathrm{N}_{2}$ asymptote.

In a subsequent paper, ${ }^{10}$ Walch and Partidge attempted a conclusive and accurate prediction of the $\mathrm{HN}_{2}$ unimolecular decomposition. They reported a systematic study of the $\mathrm{HN}_{2}$ energetics using the complete active space self-consistent field/ internally contracted configuration interaction (CASSCF/ICCI) method and the cc-pVDZ, cc-pVTZ, cc-pVQZ, and cc-pV5Z basis sets of Dunning. ${ }^{11,12}$ From such results, an extrapolation to the complete basis set limit has been obtained, which yields a barrier height of $11.34 \mathrm{kcal} / \mathrm{mol}$ and a global minimum 4.31 $\mathrm{kcal} / \mathrm{mol}$ above the $\mathrm{H}+\mathrm{N}_{2}$ asymptote. A slight difference between the constrained minimum path calculated at this level of theory and the one previously estimated using a potential energy surface ${ }^{6}$ induced those authors to conclude "...we are very confident of the theoretical lifetime predictions for the $\mathrm{HN}_{2}$ radical".

In a more recent publication, $\mathrm{Gu}$ et al. ${ }^{13}$ reported a high level CCSD(T)/aug-cc-pVQZ calculation of the stationary points for the title system. They predict the classical barrier height for the reaction $\mathrm{HN}_{2} \rightarrow \mathrm{H}+\mathrm{N}_{2}$ to be $10.7 \mathrm{kcal} / \mathrm{mol}$, thus lying 0.6 $\mathrm{kcal} / \mathrm{mol}$ below the extrapolated value reported by Walch and Partridge. ${ }^{10}$ In turn, the calculated ${ }^{13}$ exothermicity for the reaction $\mathrm{H}+\mathrm{N}_{2} \rightarrow \mathrm{HN}_{2}$ is $3.8 \mathrm{kcal} / \mathrm{mol}$, which is, thus, slightly smaller than the extrapolated value ${ }^{10}$ of $4.31 \mathrm{kcal} / \mathrm{mol}$. We may therefore argue that there is not a conclusive result yet for the $\mathrm{HN}_{2}$ lifetime.

In this work, we report the first realistic global potential energy surface for the ground electronic state of the title system, based on the double many-body expansion ${ }^{14-17}$ (DMBE) method. Being a potential fragment of larger $\mathrm{N}_{x} \mathrm{H}_{y}$ species, such as those of relevance in studying the synthesis of ammonia, it may also be of crucial importance in constructing the corresponding global DMBE forms for larger hydrogen-nitrogen polyatomic species. Indeed, this has been a major motivation for the present work. Specifically, the analytic $\mathrm{HN}_{2} \mathrm{DMBE}$ potential energy surface will be calibrated from $\sim 900 \mathrm{ab}$ initio points that were calculated at the multireference configuration interaction (MRCI) level, using the full valence complete active space (FVCAS) reference function and the aug-cc-pVQZ (AVQZ) Dunning ${ }^{11,12}$ basis set. The ab initio energies calculated in this way have been subsequently corrected, using the double 
many-body expansion-scaled external correlation ${ }^{18}$ (DMBESEC) method to extrapolate to the complete basis set/complete configuration interaction limits. As usual in DMBE theory, ${ }^{14-17}$ the potential energy surface so obtained shows the correct longrange behavior at all dissociation channels while providing a realistic representation of the surface features at all interatomic separations (e.g., van der Waals wells at intermediate atomdiatom separations).

The paper is organized as follows. Section 2 describes the $\mathrm{ab}$ initio calculations that have been performed in the present work. In Section 3, we examine the analytical representation of the potential energy surface; specifically, Section 3.1 focuses on the two-body energy terms, whereas Section 3.2 concentrates on the three-body energy terms. The main topographical features of the DMBE potential energy surface are discussed in Section 4. Some concluding remarks are given in Section 5.

\section{Ab Initio Calculations}

The ab initio calulations have been performed at the $\mathrm{MRCI}^{19}$ level, using the FVCAS $^{20}$ wave function as reference. This involves nine active orbitals $\left(7 a^{\prime}+2 a^{\prime \prime}\right)$, with the nitrogen $1 s$ orbitals forming the $1 a^{\prime}$ and $2 a^{\prime}$ molecular orbitals, having been left uncorrelated. Such a FVCAS reference wave function represents a total of 3080 configuration state functions. For the basis set, we have selected the aug-cc-pVQZ (AVQZ) of Dunning, ${ }^{11,12}$ with the correlated ab initio calculations being performed using the MOLPRO $^{21}$ package. Approximately 900 grid points have been computed to map the potential energy surface over the region defined by $1.6 \leq R_{\mathrm{N}_{2}} / a_{0} \leq 2.8,1.5 \leq$ $r_{\mathrm{H}-\mathrm{N}_{2}} / a_{0} \leq 10.0$, and $0.0 \leq \gamma \leq 90^{\circ}$ for $\mathrm{H}-\mathrm{N}_{2}$ interactions, and $1.5 \leq R_{\mathrm{NH}} / a_{0} \leq 2.4,1.5 \leq r_{\mathrm{N}-\mathrm{NH}} / a_{0} \leq 6.0$, and $0.0 \leq \gamma \leq$ $180^{\circ}$ for $\mathrm{N}-\mathrm{NH}$ interactions; $r, R$, and $\gamma$ are the corresponding atom-diatom Jacobi coordinates.

The $a b$ initio energies calculated in this way have been subsequently corrected using the DMBE-SEC ${ }^{16}$ method to account for the excitations beyond singles and doubles and, most importantly, for the incompleteness of the basis set. Thus, the total DMBE-SEC interaction energy is written as

$$
V(\boldsymbol{R})=V_{\mathrm{FVCAS}}(\boldsymbol{R})+V_{\mathrm{SEC}}(\boldsymbol{R})
$$

where

$$
\begin{aligned}
& V_{\mathrm{FVCAS}}(\boldsymbol{R})= \\
& \sum_{\mathrm{AB}} V_{\mathrm{AB}, \mathrm{FVCAS}}^{(2)}\left(R_{\mathrm{AB}}\right)+V_{\mathrm{ABC}, \mathrm{FVCAS}}^{(3)}\left(R_{\mathrm{AB}}, R_{\mathrm{BC}}, R_{\mathrm{AC}}\right)
\end{aligned}
$$

and

$$
V_{\mathrm{SEC}}(\boldsymbol{R})=\sum_{\mathrm{AB}} V_{\mathrm{AB}, \mathrm{SEC}}^{(2)}\left(R_{\mathrm{AB}}\right)+V_{\mathrm{ABC}, \mathrm{SEC}}^{(3)}\left(R_{\mathrm{AB}}, R_{\mathrm{BC}}, R_{\mathrm{AC}}\right)
$$

where $\boldsymbol{R}=\left\{\boldsymbol{R}_{i}\right\}$ is a collective variable of all internuclear distances. In turn, the first two terms of the SEC series expansion assume the form

$$
\begin{aligned}
& V_{\mathrm{AB}, \mathrm{SEC}}^{(2)}\left(R_{\mathrm{AB}}\right)=\frac{V_{\mathrm{AB}, \mathrm{FVCAS}-\mathrm{CISD}}^{(2)}\left(R_{\mathrm{AB}}\right)-V_{\mathrm{AB}, \mathrm{FVCAS}}^{(2)}\left(R_{\mathrm{AB}}\right)}{F_{\mathrm{AB}}^{(2)}}(6) \\
& \frac{V_{\mathrm{ABC}, \mathrm{SEC}}^{(3)}\left(R_{\mathrm{AB}}, R_{\mathrm{BC}}, R_{\mathrm{AC}}\right)=}{V_{\mathrm{ABC}, \mathrm{FVCAS}-\mathrm{CISD}}^{(3)}\left(R_{\mathrm{AB}}, R_{\mathrm{BC}}, R_{\mathrm{AC}}\right)-V_{\mathrm{ABC}, \mathrm{FVCAS}}^{(3)}\left(R_{\mathrm{AB}}, R_{\mathrm{BC}}, R_{\mathrm{AC}}\right)} \\
& F_{\mathrm{ABC}}^{(3)}
\end{aligned}
$$

Following previous work, ${ }^{16}$ the $F_{\mathrm{AB}}^{(2)}$ parameter in eq 6 has been chosen to reproduce the bond dissociation energy of the corresponding $\mathrm{AB}$ diatomic, while $F_{\mathrm{ABC}}^{(3)}$ has been estimated as the average of the two-body $F$-factors. Such a procedure leads to the following values: $F_{\mathrm{NN}}^{(2)}=0.758, F_{\mathrm{NH}}^{(2)}=0.948$, and $F_{\mathrm{NNH}}^{(3)}$ $=0.885$.

\section{Double Many-Body Expansion Potential Energy Surface}

3.1. Two-Body Energy Terms. The diatomic potential curves have been modeled using the extended Hartree-Fock approximate correlation energy method for diatomic molecules, including the united atom $\operatorname{limit}^{22}$ (EHFACE2U), with the available parameters being determined by fitting experimental and ab initio data. Thus, they assume the general form ${ }^{15,22}$

$$
V=V_{\mathrm{EHF}}(R)+V_{\mathrm{dc}}(R)
$$

where the label EHF refers to the extended Hartree-Fock type energy and the label dc indicates the dynamical correlation energy. As usual, the latter is modeled semiempirically by ${ }^{23}$

$$
V_{\mathrm{dc}}(R)=-\sum_{n=6,8,10} C_{n} \chi_{n}(R) R^{-n}
$$

with the damping function for the dispersion coefficients assuming the form

$$
\chi_{n}(R)=\left[1-\exp \left(-A_{n} \frac{R}{\rho}-B_{n} \frac{R^{2}}{\rho^{2}}\right)\right]^{n}
$$

In eq $10, A_{n}$ and $B_{n}$ are auxiliary functions that are defined by

$$
\begin{gathered}
A_{n}=\alpha_{0} n^{-\alpha_{1}} \\
B_{n}=\beta_{0} \exp \left(-\beta_{1} n\right)
\end{gathered}
$$

where $\alpha_{0}, \beta_{0}, \alpha_{1}$, and $\beta_{1}$ are universal dimensionless parameters for all isotropic interactions: ${ }^{14,15} \alpha_{0}=16.36606, \alpha_{1}=0.70172$, $\beta_{0}=17.19338$, and $\beta_{1}=0.09574$. In turn, the scaling parameter $\rho$ is defined as

$$
\rho=5.5+1.25 R_{0}
$$

where $R_{0}=2\left(\left\langle r_{\mathrm{A}}^{2}\right\rangle^{1 / 2}+\left\langle r_{\mathrm{B}}^{2}\right\rangle^{1 / 2}\right)$ is the LeRoy ${ }^{24}$ parameter for onset the undamped $R^{-n}$ expansion, and $\left\langle r_{\mathrm{X}}^{2}\right\rangle$ is the expectation value of the squared radius for the outermost electrons of atom $\mathrm{X}$ (where $\mathrm{X}=\mathrm{A}, \mathrm{B}$ ). Finally, the exponential decaying portion of the EHF-type energy term assumes the general form

$$
V_{\mathrm{EHF}}(R)=-\frac{D}{R}\left(1+\sum_{i=1}^{n} a_{i} r^{i}\right) \exp (-\gamma r)
$$

where

$$
\gamma=\gamma_{0}\left[1+\gamma_{1} \tanh \left(\gamma_{2} r\right)\right]
$$

$r=R-R_{e}$ is the displacement from the equilibrium diatomic geometry, and $D$ and $a_{i}(i=1, \ldots, n)$ are adjustable parameters to be obtained as described elsewhere. ${ }^{15,22}$ Specifically, the potential curve of ground-state $\mathrm{N}_{2}\left(\mathrm{X}^{1} \Sigma^{+}\right)$has been calibrated by fitting simultaneously vibrational frequencies, RydbergKleing-Rees (RKR) turning points, ${ }^{25}$ and the ab initio energies calculated in the present work for the repulsive wing. Figure 1 shows that the final potential curve reproduces our calculated 


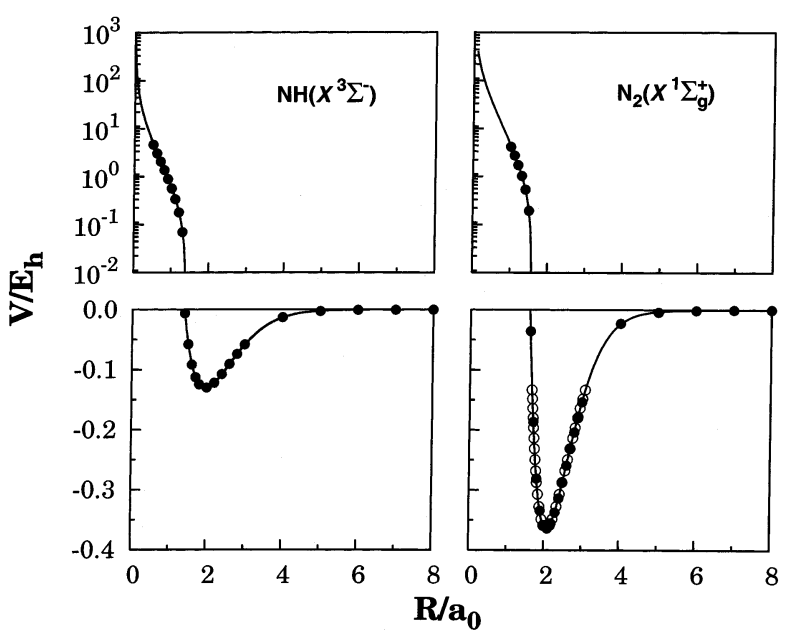

Figure 1. EHFACE2U potential energy curves for $\mathrm{NH}\left({ }^{3} \Sigma^{-}\right)$and $\mathrm{N}_{2}\left({ }^{1} \sum_{\mathrm{g}}\right.$ ). Key for symbols: (O) Rydberg-Kleing-Ress (RKR) data and (๑) ab initio points.

TABLE 1: Parameters in the Two-Body Energy Curves

\begin{tabular}{lll}
\hline & $\mathrm{NH}\left(\mathrm{X}^{3} \Sigma^{-}\right)$ & \multicolumn{1}{c}{$\mathrm{N}_{2}\left(\mathrm{X}^{1} \Sigma_{\mathrm{g}}^{+}\right)$} \\
\hline$R_{\mathrm{e}}\left(a_{0}\right)$ & 1.9650 & 2.0743 \\
$D\left(E_{\mathrm{h}}\right)$ & 0.22903401 & 0.70211920 \\
$a_{1}\left(a_{0}^{-1}\right)$ & 2.14664173 & 2.21167295 \\
$a_{2}\left(a_{0}^{-2}\right)$ & 0.84471252 & 0.99148545 \\
$a_{3}\left(a_{0}^{-3}\right)$ & 0.52590829 & 1.55376929 \\
$\gamma_{0}\left(a_{0}^{-1}\right)$ & 1.563792 & 1.678669 \\
$\gamma_{1}\left(a_{0}^{-1}\right)$ & 0.661116 & 0.674118 \\
$\gamma_{2}\left(a_{0}^{-1}\right)$ & 0.282985 & 0.716957 \\
$R_{0}\left(a_{0}\right)$ & 6.7560 & 6.5938 \\
$C_{6} /\left(E_{\mathrm{h}} a_{0}^{-6}\right)$ & 12.27 & 22.8 \\
$C_{8} /\left(E_{\mathrm{h}} a_{0}^{-8}\right)$ & 232.6 & 442.6 \\
$C_{10} /\left(E_{\mathrm{h}} a_{0}^{-10}\right)$ & 5775 & 9722
\end{tabular}

$\mathrm{ab}$ initio energies. In turn, for ground-state imidogen, $\mathrm{NH}\left(\mathrm{X}^{3} \Sigma^{-}\right)$, we have used a previously reported potential energy curve ${ }^{26}$ calibrated from the MRCI+Q ab initio points of Stallcop et al. ${ }^{27}$ The numerical values of all the parameters are, for both diatomic potentials, gathered in Table 1 .

3.2. Three-Body Energy Terms. In regard to the two-body energies, the three-body energy splits into several contributions. First, the three-body energy is divided into an extended HartreeFock part and a dynamical correlation part. For the three-body dynamical correlation, we select the form that was proposed elsewhere by one of us $^{28}$ for the water molecule, namely

$$
V_{\mathrm{dc}}^{(3)}=\sum_{i} \sum_{n} f_{i}(\boldsymbol{R}) C_{n}^{(i)}\left(R_{i}, \theta_{i}\right) \chi_{n}\left(r_{i}\right) r_{i}^{-n}
$$

where the $i$ label represents the $\mathrm{I}-\mathrm{JK}$ channel associated with the center of mass separation $r_{i}, R_{i}$ is the $\mathrm{J}-\mathrm{K}$ bond distance, and $\cos \theta_{i}=\vec{r}_{i} \vec{R}_{i} /\left|\vec{r}_{i} \vec{R}_{i}\right|$. (For the notation, see Figure 1 of ref 16.) For $n=6,8$, and $10, C_{n}^{(i)}\left(R_{i}, \theta_{i}\right)$ are atom-diatom dispersion coefficients, which are given by

$$
C_{n}^{(i)}=\sum_{L} C_{n}^{L} P_{L}\left(\cos \theta_{i}\right)
$$

where $P_{L}\left(\cos \theta_{i}\right)$ denotes the $L$-th term of the Legendre polynomial expansion. In addition, the function $\chi_{n}\left(r_{i}\right)$ in eq 16 is the corresponding diatomic damping function. Moreover, $f_{i}(\boldsymbol{R})$ is a switching function that is chosen from the requirement that its value must be +1 at $R_{i}=R_{i}^{e}$ and $r_{i} \rightarrow \infty$, and 0 when $R_{i} \rightarrow$ $\infty$. One such form is ${ }^{28}$

$$
f_{i}=\frac{1}{2}\left\{1-\tanh \left[\xi\left(\eta s_{i}-s_{j}-s_{k}\right)\right]\right\}
$$

where $s_{i}=R_{i}-R_{i}^{e}$ (corresponding expressions apply to $s_{j}, s_{k}$, $f_{j}$, and $f_{k}$ ) and $\eta$ is a constant that is chosen to ensure the proper asymptotic behavior. Following previous work, ${ }^{28}$ we adopt a value of $\eta=3$. In addition, the parameter $\xi$ has been assumed to be equal to $1.0 a_{0}^{-1}$. Regarding the damping function $\chi_{n}\left(r_{i}\right)$, we still adopt eq 10 but replace $R$ by the center-of-mass separation for the relevant atom-diatom channel. In addition, the value of $\rho$ has been assumed by considering $R_{0}$ in eq 13 as the average of the LeRoy parameters for $\mathrm{NO}$ and $\mathrm{SiH}$, which leads to a value of $\rho=15.9$.

3.3. Three-Body Dynamical Correlation Energy. The $L=$ 0,2 , and 4 components of the atom-diatom dispersion coefficients have been considered, with the involved internuclear dependences being estimated as reported elesewhere, ${ }^{29}$ i.e., using the dipolar polarizabilities calculated in the present work at the MRCI level of theory jointly with the generalized SlaterKirkwood approximation. ${ }^{30}$ The atom-diatom dispersion coefficients thus calculated were then fitted to the form

$$
\begin{aligned}
& C_{n}^{\mathrm{A}-\mathrm{BC}}(R)= \\
& \quad C_{n}^{\mathrm{AB}}+C_{n}^{\mathrm{AC}}+D_{\mathrm{M}}\left(1+\sum_{i=1}^{3} a_{i} r^{i}\right) \exp \left(-a_{1} r-\sum_{i=2}^{3} b_{i} r^{i}\right)
\end{aligned}
$$

where $r=R-R_{\mathrm{M}}$ is the displacement relative to the position of the maximum. The parameters that resulted from such fits are reported in Table 2, whereas the internuclear dependences of the dispersion coefficients are shown in Figure 2.

As noted elsewhere, ${ }^{28}$ eq 16 causes an overestimation of the dynamical correlation energy at the atom-diatom dissociation channels. To correct such a behavior, we have multiplied the two-body dynamical correlation energy for the $i$-th pair by $f_{i}(\boldsymbol{R})$ and correspondingly for channels $j$ and $k$. This ensures ${ }^{28}$ that the only two-body contribution at the $i$-th channel is that of JK.

3.4. Three-Body Extended Hartree-Fock Energy. By removing, for a given triatomic geometry, the sum of the twobody energy terms from the corresponding DMBE-SEC interaction energies (eq 3), which was defined with respect to the infinitely separated ground-state atoms, one obtains the total three-body energy. Therefore, by subtracting the three-body dynamical correlation contribution (eq 16) from the total threebody energy that is calculated in that way, one obtains the threebody extended Hartree-Fock energy contribution. To represent the latter, we use the following three-body distributedpolynomial $^{31}$ form:

$V_{\mathrm{EHF}}^{(3)}=\sum_{j=1}^{5} P^{(j)}\left(Q_{1}, Q_{2}, Q_{3}\right) \prod_{i=1}^{3}\left\{1-\tanh \left[\gamma_{i}^{j}\left(R_{i}-R_{i}^{j, \mathrm{ref}}\right)\right]\right\}$

where $P^{(j)}\left(Q_{1}, Q_{2}, Q_{3}\right)$ is the $j$-th polynomial. To the sixth order, this assumes the form 


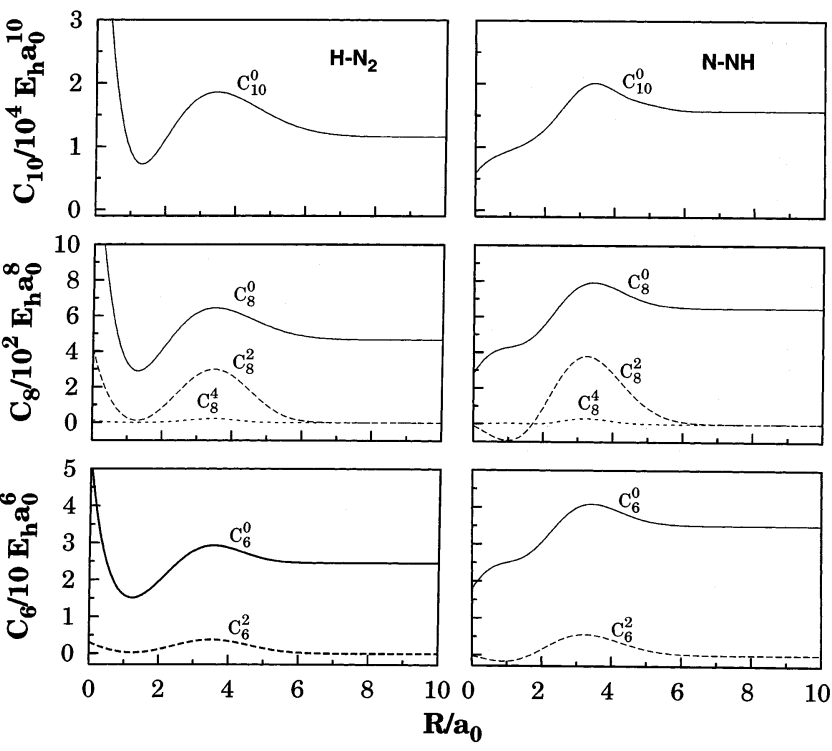

Figure 2. Dispersion coefficients for atom-diatom asymptotic channels of $\mathrm{HN}_{2}$, as a function of the corresponding internuclear distance $R$.

$$
\begin{gathered}
P^{j}\left(Q_{1}, Q_{2}, Q_{3}\right)=c_{1}+c_{2} Q_{1}+c_{3} Q_{3}+c_{4} Q_{1}^{2}+c_{5} S_{2 a}^{2}+ \\
c_{6} Q_{1} Q_{3}+c_{7} S_{2 b}^{2}+c_{8} Q_{1}^{3}+c_{9} Q_{1} S_{2 a}^{2}+c_{10} S_{3}^{3}+c_{11} Q_{1}^{2} Q_{3}+ \\
c_{12} Q_{1} S_{2 b}^{2}+c_{13} Q_{3} S_{2 a}^{2}+c_{14} Q_{1}^{4}+c_{15} Q_{1}^{2} S_{2 a}^{2}+c_{16} S_{2 a}^{4}+ \\
c_{17} Q_{1} S_{3}^{3}+c_{18} Q_{1}^{3} Q_{3}+c_{19} Q_{1}^{2} S_{2 b}^{2}+c_{20} Q_{1} Q_{3} S_{2 a}^{2}+c_{21} Q_{3} S_{3}^{3}+ \\
c_{22} S_{2 a}^{2} S_{2 b}^{2}+c_{23} Q_{1}^{5}+c_{24} Q_{1}^{3} S_{2 a}^{2}+c_{25} Q_{1} S_{2 a}^{4}+c_{26} Q_{1}^{2} S_{3}^{3}+ \\
c_{27} S_{2 a}^{2} S_{3}^{3}+c_{28} Q_{1}^{4} Q_{3}+c_{29} Q_{1}^{3} S_{2 b}^{2}+c_{30} Q_{1}^{2} Q_{3} S_{2 a}^{2}+ \\
c_{31} Q_{1} Q_{3} S_{3}^{3}+c_{32} Q_{1} S_{2 a}^{2} S_{2 b}^{2}+c_{33} Q_{3} S_{2 a}^{4}+c_{34} S_{2 b}^{2} S_{3}^{3}+c_{35} Q_{1}^{6}+ \\
c_{36} Q_{1}^{4} S_{2 a}^{2}+c_{37} Q_{1}^{2} S_{2 a}^{4}+c_{38} Q_{1}^{3} S_{3}^{3}+c_{39} Q_{1} S_{2 a}^{2} S_{3}^{3}+c_{40} S_{2 a}^{6}+ \\
c_{41} S_{3}^{6}+c_{42} Q_{1}^{5} Q_{3}+c_{43} Q_{1}^{4} S_{2 b}^{2}+c_{44} Q_{1}^{3} Q_{3} S_{2 a}^{2}+c_{45} Q_{1}^{2} Q_{3} S_{3}^{3}+ \\
c_{46} Q_{1}^{2} S_{2 a}^{2} S_{2 b}^{2}+c_{47} Q_{1} Q_{3} S_{2 a}^{4}+c_{48} Q_{1} S_{2 b}^{2} S_{3}^{3}+c_{49} Q_{3} S_{2 a}^{2} S_{3}^{3}+ \\
c_{50} S_{2 a}^{4} S_{2 b}^{2}(21)
\end{gathered}
$$

where $S_{2 a}^{2}=Q_{2}^{2}+Q_{3}^{2}, S_{2 b}^{2}=Q_{2}^{2}-Q_{3}^{2}$, and $S_{3}^{3}=Q_{3}^{3}-3 Q_{2}^{2} Q_{3}$, with $Q_{i}(i=1-3)$ being symmetry coordinates that are defined, for the $j$-th polynomial, by

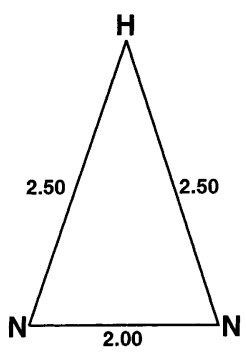

a)

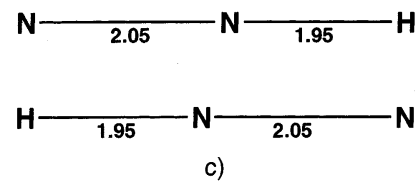

Figure 3. Reference geometries (with distances in atomic units, a.u.) for the three-body EHF portion of the potential energy surface. See text for details.

$$
\left(\begin{array}{l}
Q_{1} \\
Q_{2} \\
Q_{3}
\end{array}\right)=\left(\begin{array}{ccc}
\sqrt{\frac{1}{3}} & \sqrt{\frac{1}{3}} & \sqrt{\frac{1}{3}} \\
0 & \sqrt{\frac{1}{2}} & -\sqrt{\frac{1}{2}} \\
\sqrt{\frac{2}{3}} & -\sqrt{\frac{1}{6}} & -\sqrt{\frac{1}{6}}
\end{array}\right)\left(\begin{array}{l}
R_{1}-R_{1}^{j, \text { ref }} \\
R_{2}-R_{2}^{j, \text { ref }} \\
R_{3}-R_{3}^{j, \text { ref }}
\end{array}\right)
$$

where the superscript "ref" means a reference geometry. Figure 3 displays the reference geometries that are used to define the displacement coordinates used to write the five EHF-type polynomials in eq 20 . Note that the first and second polynomials

\begin{tabular}{|c|c|c|c|c|c|c|}
\hline & $C_{6}^{0}(R)$ & $C_{6}^{2}(R)$ & $C_{8}^{0}(R)$ & $C_{8}^{2}(R)$ & $C_{8}^{4}(R)$ & $C_{10}^{0}(R)$ \\
\hline \multicolumn{7}{|c|}{$\mathrm{H}-\mathrm{N}_{2}$} \\
\hline$R_{\mathrm{M}}\left(a_{0}\right)$ & 3.5667 & 3.5090 & 3.5026 & 3.4863 & 3.4870 & 3.4608 \\
\hline$D_{\mathrm{M}}\left(E_{\mathrm{h}}\right)$ & 4.7294 & 3.7419 & 178.7058 & 298.9732 & 22.9832 & 7028.8116 \\
\hline$a_{1}\left(a_{0}^{-1}\right)$ & 1.27779982 & 0.85158213 & 1.13108335 & 0.88460242 & 0.87930621 & 1.09018628 \\
\hline$a_{2}\left(a_{0}^{-2}\right)$ & 0.30041055 & 0.18434994 & 0.27793909 & 0.19656342 & 0.19895365 & 0.27579487 \\
\hline$a_{3}\left(a_{0}^{-3}\right)$ & -0.00310054 & -0.00096988 & -0.00010674 & -0.00093354 & 0.00096552 & 0.00170486 \\
\hline$b_{2}\left(a_{0}^{-2}\right)$ & 0.31567776 & 0.26909843 & 0.13571843 & 0.29319575 & 0.94526128 & 0.09608657 \\
\hline$b_{3}\left(a_{0}^{-3}\right)$ & 0.04787065 & 0.02884454 & 0.00997824 & 0.04431067 & 0.21637709 & 0.00694565 \\
\hline \multicolumn{7}{|c|}{$\mathrm{N}-\mathrm{NH}$} \\
\hline$R_{\mathrm{M}}\left(a_{0}\right)$ & 3.4400 & 3.2316 & 3.4371 & 3.2506 & 3.2187 & 3.4352 \\
\hline$D_{\mathrm{M}}\left(E_{\mathrm{h}}\right)$ & 5.8549 & 5.5978 & 143.9529 & 379.7076 & 31.0459 & 4394.6574 \\
\hline$a_{1}\left(a_{0}^{-1}\right)$ & 1.59525394 & 1.02648421 & 1.58449587 & 0.93156640 & 1.64450586 & -0.07374322 \\
\hline$a_{2}\left(a_{0}^{-2}\right)$ & 0.70875804 & 0.27083216 & 0.70365110 & 0.18277832 & 0.86551327 & -0.60710628 \\
\hline$a_{3}\left(a_{0}^{-3}\right)$ & 0.10447827 & 0.01598388 & 0.10352265 & -0.00162653 & 0.13961200 & 0.49713866 \\
\hline$b_{2}\left(a_{0}^{-2}\right)$ & 0.28481314 & 0.27023430 & 0.28978070 & 0.31873743 & 0.68479326 & 0.55319506 \\
\hline$b_{3}\left(a_{0}^{-3}\right)$ & $1.0 \times 10^{-8}$ & $1.0 \times 10^{-8}$ & $1.0 \times 10^{-8}$ & $1.0 \times 10^{-8}$ & $1.0 \times 10^{-8}$ & 0.10683628 \\
\hline
\end{tabular}
(which refer to the first and second sets of coefficients in Table 3 ) are respectively centered in structures a) and b) of Figure 3. In turn, two other polynomials use the same set of coefficients (the third column of Table 3 ), which are centered in structure c) of Figure 3. The last polynomial, $P^{(5)}\left(Q_{1}, Q_{2}, Q_{3}\right)$, is centered at reference geometry d) in Figure 3 , which lies close to the equilibrium structure of the weakly bound $\mathrm{H} \cdots \mathrm{N}_{2}$ van der Waals species. Note that this includes up to cubic terms only, because this proved to be sufficient for our fitting purposes. To obtain $R_{i}^{j, \text { ref }}$, we have first assumed their values to coincide with the

TABLE 2: Numerical Values of the Parameters in eq 19 
TABLE 3: Numerical Values of the Extended

Hartree-Fock Energy (eq 20)

\begin{tabular}{|c|c|c|c|c|}
\hline coefficient & $P^{(1)}$ & $P^{(2)}$ & $P^{(3)}+P^{(4)}$ & $P^{(5)}$ \\
\hline$\overline{c_{1}\left(a_{0}^{0}\right)}$ & -35.35722711 & 50.89357137 & 0.64204663 & -0.00037787 \\
\hline$c_{2}\left(a_{0}^{-1}\right)$ & 1.43122305 & -8.20511664 & -1.57901897 & -0.00190090 \\
\hline$c_{3}\left(a_{0}^{-1}\right)$ & -9.87617675 & -44.75381891 & -0.94137581 & -0.00261274 \\
\hline$c_{4}\left(a_{0}^{-2}\right)$ & -18.80416915 & 26.48543000 & 1.81933025 & 0.00029084 \\
\hline$c_{5}\left(a_{0}^{-2}\right)$ & -21.78867083 & 32.57491690 & 0.60735250 & -0.00057451 \\
\hline$c_{6}\left(a_{0}^{-2}\right)$ & -11.07940674 & -33.13037794 & 0.52335000 & -0.00038871 \\
\hline$c_{7}\left(a_{0}^{-2}\right)$ & 5.01128977 & 5.08772061 & 0.25855378 & 0.00055106 \\
\hline$c_{8}\left(a_{0}^{-3}\right)$ & 0.96613480 & -3.99536563 & -1.18504903 & 0. \\
\hline$c_{9}\left(a_{0}^{-3}\right)$ & -0.77697807 & 10.11891334 & -0.63735021 & -0.00076654 \\
\hline$c_{10}\left(a_{0}^{-3}\right)$ & -0.92099871 & 4.10517993 & 0.12413155 & 0. \\
\hline$c_{11}\left(a_{0}^{-3}\right)$ & -6.01375589 & -16.78809143 & -0.38833222 & -0.00104352 \\
\hline$c_{12}\left(a_{0}^{-3}\right)$ & 0.90509055 & -16.20351814 & 0.11928222 & 0.00074255 \\
\hline$c_{13}\left(a_{0}^{-3}\right)$ & -514061355 & -17.17930402 & -0.82128727 & 0. \\
\hline$c_{14}\left(a_{0}^{-4}\right)$ & -3.40765945 & 3.78922878 & 0.63973511 & 0. \\
\hline$c_{15}\left(a_{0}^{-4}\right)$ & -1.88077926 & 3.13322630 & -0.26288962 & 0. \\
\hline$c_{16}\left(a_{0}^{-4}\right)$ & -2.68379376 & 5.69616150 & 0.17067403 & 0. \\
\hline$c_{17}\left(a_{0}^{-4}\right)$ & 4.45588653 & -5.81699928 & -0.18560723 & 0. \\
\hline$c_{18}\left(a_{0}^{-4}\right)$ & -4.05543497 & -7.84835242 & 0.12054093 & 0. \\
\hline$c_{19}\left(a_{0}^{-4}\right)$ & 1.70217322 & -11.08806364 & -0.17812786 & 0. \\
\hline$c_{20}\left(a_{0}^{-4}\right)$ & -3.39696345 & -5.64359911 & 0.34470547 & 0. \\
\hline$c_{21}\left(a_{0}^{-4}\right)$ & 0.52809629 & 1.62229042 & -0.24358630 & 0. \\
\hline$c_{22}\left(a_{0}^{-4}\right)$ & 0.96757611 & 1.49907056 & 0.29036290 & 0. \\
\hline$c_{23}\left(a_{0}^{-5}\right)$ & 0.07042292 & -0.52463644 & -0.18613603 & 0. \\
\hline$c_{24}\left(a_{0}^{-5}\right)$ & -1.13489314 & 2.85006561 & 0.39057365 & 0. \\
\hline$c_{25}\left(a_{0}^{-5}\right)$ & 1.21500520 & 0.84486292 & -0.10864732 & 0. \\
\hline$c_{26}\left(a_{0}^{-5}\right)$ & 0.90416261 & -3.39970612 & -0.08699980 & 0. \\
\hline$c_{27}\left(a_{0}^{-5}\right)$ & -0.37424676 & 0.07267052 & -0.09095443 & 0. \\
\hline$c_{28}\left(a_{0}^{-5}\right)$ & -1.41649184 & -0.91682855 & -0.10631151 & 0. \\
\hline$c_{29}\left(a_{0}^{-5}\right)$ & -0.04756769 & -1.68254925 & 0.26417945 & 0. \\
\hline$c_{30}\left(a_{0}^{-5}\right)$ & 0.61305975 & -3.72605017 & 0.33025474 & 0. \\
\hline$c_{31}\left(a_{0}^{-5}\right)$ & 1.84502268 & 3.16507583 & 0.00837542 & 0. \\
\hline$c_{32}\left(a_{0}^{-5}\right)$ & -0.58400452 & -1.42401824 & -0.07300291 & 0. \\
\hline$c_{33}\left(a_{0}^{-5}\right)$ & -0.13609210 & -2.12175750 & -0.21211357 & 0. \\
\hline$c_{34}\left(a_{0}^{-5}\right)$ & -0.05015587 & 1.04011399 & 0.23138290 & 0. \\
\hline$c_{35}\left(a_{0}^{-6}\right)$ & -0.12738976 & 0.02661556 & 0.03395371 & 0. \\
\hline$c_{36}\left(a_{0}^{-6}\right)$ & 0.62237114 & -0.02032757 & -0.18379018 & 0. \\
\hline$c_{37}\left(a_{0}^{-6}\right)$ & 0.10324812 & 0.78316160 & 0.05864884 & 0. \\
\hline$c_{38}\left(a_{0}^{-6}\right)$ & -0.42328134 & -0.45490030 & -0.05717854 & 0. \\
\hline$c_{39}\left(a_{0}^{-6}\right)$ & 0.76664528 & -0.77298638 & 0.03477564 & 0. \\
\hline$c_{40}\left(a_{0}^{-6}\right)$ & -0.21436778 & 0.45857204 & 0.00603242 & 0. \\
\hline$c_{41}\left(a_{0}^{-6}\right)$ & -0.03021494 & 0.16268811 & -0.04950996 & 0. \\
\hline$c_{42}\left(a_{0}^{-6}\right)$ & -0.17424122 & 0.08238406 & -0.03113967 & 0. \\
\hline$c_{43}\left(a_{0}^{-6}\right)$ & 0.10050895 & -0.35417930 & 0.04913502 & 0. \\
\hline$c_{44}\left(a_{0}^{-6}\right)$ & 0.61827911 & -1.07342606 & -0.00694684 & 0. \\
\hline$c_{45}\left(a_{0}^{-6}\right)$ & 0.42896861 & 1.11079450 & 0.04085605 & 0. \\
\hline$c_{46}\left(a_{0}^{-6}\right)$ & -0.37356092 & -0.70735541 & -0.27048435 & 0. \\
\hline$c_{47}\left(a_{0}^{-6}\right)$ & 0.29520788 & -1.15920924 & -0.04881234 & 0. \\
\hline$c_{48}\left(a_{0}^{-6}\right)$ & -0.37797059 & 0.53374300 & -0.18376155 & 0. \\
\hline$c_{49}\left(a_{0}^{-6}\right)$ & 0.23373482 & 0.20831280 & 0.02121087 & 0. \\
\hline$c_{50}\left(a_{0}^{-6}\right)$ & 0.16170869 & -0.34419861 & 0.15943928 & 0. \\
\hline$\gamma_{1}^{j}\left(a_{0}^{-1}\right)$ & 1.30 & 0.40 & 1.55 & 1.00 \\
\hline$\gamma_{2}^{j}\left(a_{0}^{-1}\right)$ & 1.10 & 1.35 & $-0.55^{a}$ & 0.35 \\
\hline$\gamma_{3}^{j}\left(a_{0}^{-1}\right)$ & 1.10 & 1.35 & $1.55^{a}$ & 0.35 \\
\hline$R_{1}^{j, \text { ref }}\left(a_{0}\right)$ & 2.00 & 2.60 & 2.05 & 2.08 \\
\hline$R_{2}^{j, \text { ref }}\left(a_{0}\right)$ & 2.50 & 2.05 & $1.95^{b}$ & 7.00 \\
\hline$R_{3}^{j, \text { ref }}\left(a_{0}\right)$ & 2.50 & 2.05 & $4.00^{b}$ & 7.00 \\
\hline
\end{tabular}

${ }^{a}$ The values indicated should be interchanged when writing polynomials $P^{3}$ and $P^{4}$. ${ }^{b}$ See footnote a.

bond distances of the associated stationary points, and, subsequently, relaxed this condition via a trial-and-error least-squares

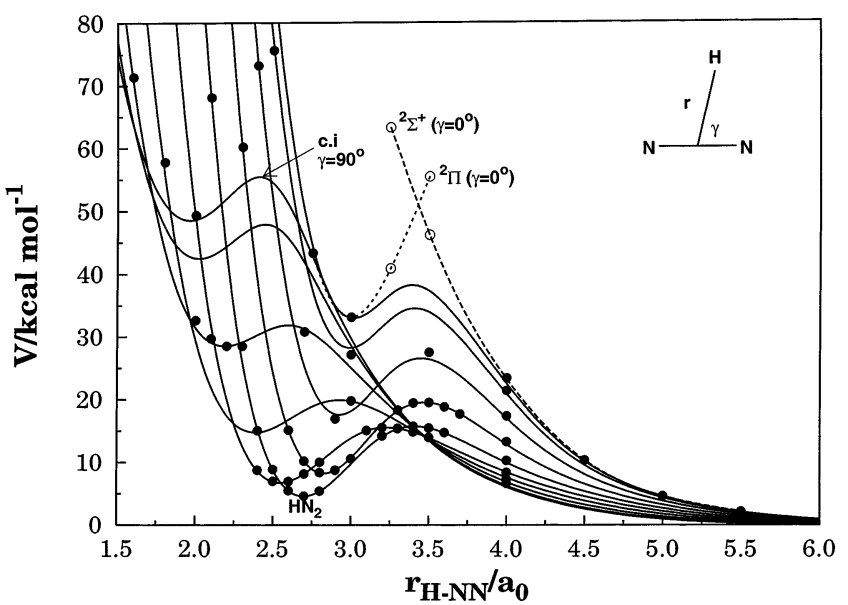

Figure 4. Selected cuts of the $\mathrm{HN}_{2}$ potential energy surface. The figure shows 10 minimum energy cuts for fixed values of $\gamma$ as a function of the distance $r_{\mathrm{H}-\mathrm{N}_{2}}$. Note the avoided crossing for T-shaped and linear configurations. Unfilled points correspond to geometries close to the conical intersection, which have not been included in the least-squares fitting procedure.

TABLE 4: Stratified Root-Mean-Square Deviations

\begin{tabular}{cccc}
\hline $\begin{array}{c}\text { energy } \\
(\mathrm{kcal} / \mathrm{mol})\end{array}$ & number of points & $\begin{array}{c}\text { maximum deviation } \\
(\mathrm{kcal} / \mathrm{mol})\end{array}$ & $\mathrm{rmsd}^{a}$ \\
\hline 20 & 224 & 0.90 & 0.152 \\
40 & 308 & 2.36 & 0.256 \\
60 & 540 & 9.06 & 0.620 \\
80 & 570 & 9.06 & 0.714 \\
100 & 597 & 9.06 & 0.765 \\
200 & 825 & 10.06 & 0.935 \\
300 & 887 & 10.06 & 0.982 \\
500 & 913 & 10.06 & 1.070
\end{tabular}

${ }^{a}$ Root-mean-square deviation.

fitting procedure. The nonlinear range-determining parameters $\gamma_{i}^{j}$ have been optimized in a similar way. Thus, the complete set of parameters totals 160 linear coefficients $c_{i}$, 15 nonlinear coefficients $\gamma_{i}^{j}$, and 15 reference geometries $R_{i}^{j, \text { ref }}$. Their optimal numerical values are collected in Table 3. A total of 913 points have been used for the calibration procedure, with the energies covering a range up to $500 \mathrm{kcal} / \mathrm{mol}$ above the $\mathrm{H}+$ $\mathrm{N}_{2}$ asymptote.

Table 4 shows the stratified root-mean-square deviation (rmsd) values of the final potential energy surface, with respect to the fitted and nonfitted ab initio energies. This table shows that the final potential energy surface is able to fit the region of major chemical interest (308 ab initio points with energies up to $40 \mathrm{kcal} / \mathrm{mol})$ with a high accuracy $(\mathrm{rmsd}=0.256 \mathrm{kcal} / \mathrm{mol})$. This region includes the global minimum and the transition state for the $\mathrm{H}+\mathrm{N}_{2}$ dissociation process. The major deviations correspond to points close to the conical intersection (thus, they have not been included in the fitting procedure, to warrant a behavior of the final single-valued form that is as smooth as possible) and at high repulsive regions of the potential surface. Clearly, the DMBE potential energy surface that has been obtained in this way accurately reproduces all fitted and nonfitted ab initio points, up to $500 \mathrm{kcal} / \mathrm{mol}$.

Figure 4 illustrates the quality of the final fitted form. The profiles correspond to minimum energy cuts for 10 distinct values of $\gamma$, whereas the displayed ab initio points form a small set of the total number of points used in the fitting procedure; they have been calculated by optimizing $R_{\mathrm{N}_{2}}$ at specified values of $\gamma$ and $r_{\mathrm{H}-\mathrm{N}_{2}}$. The two conical intersections that result for $\mathrm{T}$-shaped and linear configurations are clearly visible in Figure 


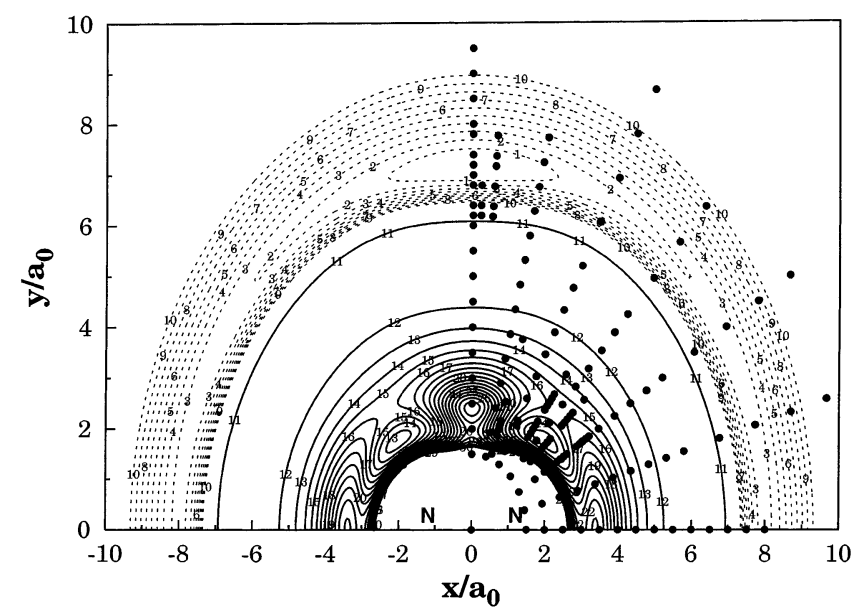

Figure 5. Isoenergy contour plot for a $\mathrm{H}$ atom moving around a partially relaxed $\mathrm{N}_{2}$ diatomic $\left(1.8 a_{0} \leq R_{\mathrm{N}_{2}} \leq 2.4 a_{0}\right)$, which lies along the $x$-axis with the center of the bond fixed at the origin. Contours are equally spaced by $0.005 E_{\mathrm{h}}$, starting at $-0.3640 E_{\mathrm{h}}$. Contours in dashed lines emphasize the van der Waals minimum; they are equally spaced by $0.000005 E_{\mathrm{h}}$, starting at $-0.36414 E_{\mathrm{h}}$. Also shown are the calculated ab initio points for the $\mathrm{H}$ atom moving around $\mathrm{N}_{2}$ diatomic, whose bond distance varied over a range from $1.6 a_{0}$ to $2.8 a_{0}$.

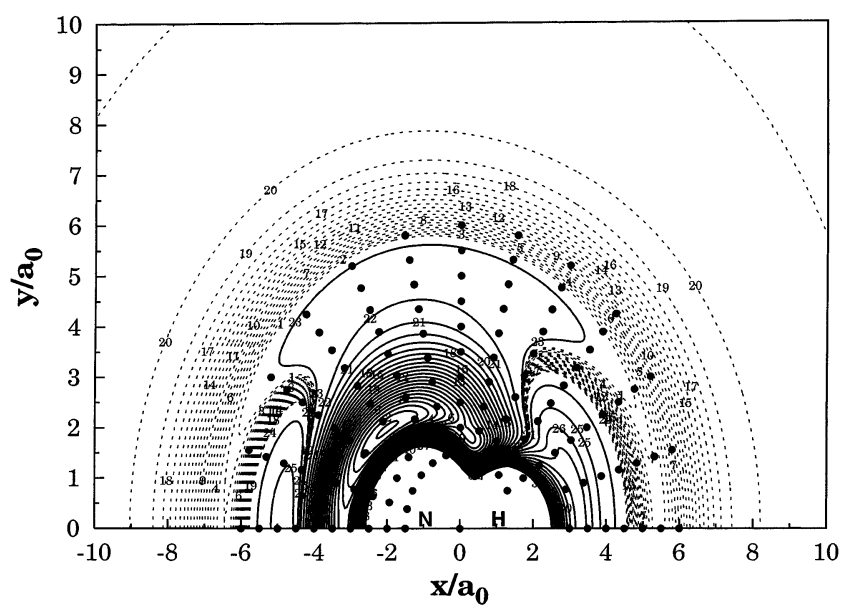

Figure 6. Isoenergy contour plot for a $\mathrm{N}$ atom moving around a partially relaxed NH diatomic $\left(1.8 a_{0} \leq R_{\mathrm{NH}} \leq 2.3 a_{0}\right)$, which lies along the $x$-axis with the center of the bond fixed at the origin. Contours are equally spaced by $0.01 E_{\mathrm{h}}$, starting at $-0.355 E_{\mathrm{h}}$. The contours in dashed are equally spaced by $0.0002 E_{\mathrm{h}}$, starting at $-0.1340 E_{\mathrm{h}}$. Also shown are the calculated ab initio points for the $\mathrm{N}$ atom around a $\mathrm{NH}$ diatomic, whose bond distance varied over a range from $1.5 a_{0}$ to $2.4 a_{0}$.

4. We emphasize that the regions close to such topological features are smoothed out (i.e., they resemble avoided intersections) in the single-valued representation used in the present work. Thus, the points close to the actual crossing seam (indicated in the plot as unfilled circles) have not been included in the fitting procedure, to avoid spoiling its quality at the regions of more chemical interest.

\section{Major Features of Potential Energy Surface}

Figures 5 and 6 illustrate the major topographical features of the $\mathrm{HN}_{2}$ DMBE potential energy surface that has been calculated in the present work. Specifically, Figure 5 shows energy contours for $\mathrm{a} \mathrm{H}$ atom moving around a $\mathrm{N}_{2}$ ground-state diatomic whose bond length is partially relaxed for each position of the $\mathrm{H}$ atom. The corresponding plot for a $\mathrm{N}$ atom moving around $\mathrm{NH}$ is presented in Figure 6. The salient features from these plots are some of the most relevant stationary points for the

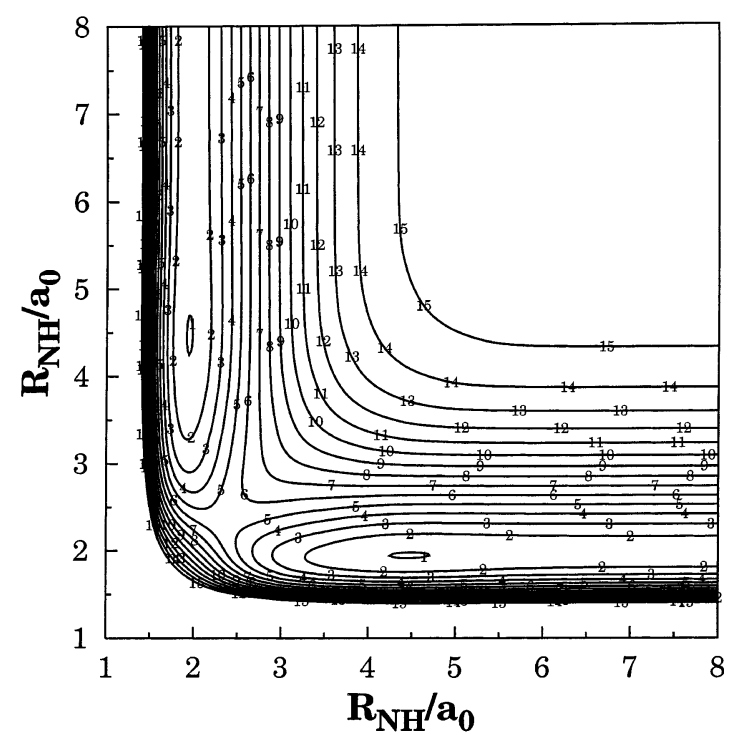

Figure 7. Isoenergy contour plot for the stretching of the two $\mathrm{NH}$ bonds in linear $\mathrm{N}-\mathrm{H}-\mathrm{N}$. Contours are equally spaced by $0.009 E_{\mathrm{h}}$, starting at $-0.1335 E_{\mathrm{h}}$. The two equivalent structures $\mathrm{NH} \cdots \mathrm{N}$ and $\mathrm{N} \cdot \cdot \mathrm{HN}$ indicate linear transition states, which appear as hydrogenbonded minima, with respect to the stretching coordinate (see text).

TABLE 5: Stationary Points of the DMBE Potential Energy Surface, for Various Features

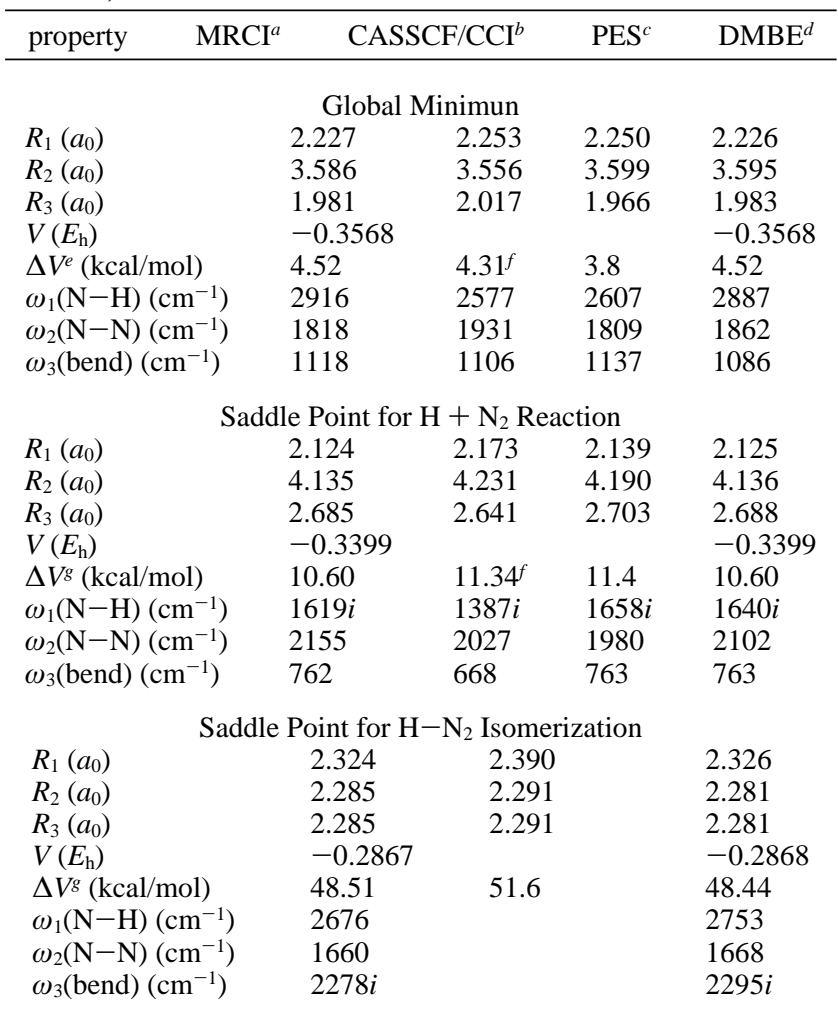

${ }^{a}$ From this work, from a fit to a Taylor-series-type expansion around the stationary point; see text. ${ }^{b}$ From ref $8 .{ }^{c}$ From ref $6 .{ }^{d}$ This work. ${ }^{e}$ Relative to the $\mathrm{H}+\mathrm{N}_{2}$ asymptote. ${ }^{f}$ From ref 10 ; value extrapolated to the complete basis set limit. ${ }^{g}$ Relative to the global minimum.

title system. A characterization of their attributes (geometry, energy, and vibrational frequencies) is reported in Table 5. Also included for comparison in this table are the results that have been obtained from other potential energy surfaces. Note that we have calculated a denser grid of energies in the vicinity of some stationary points which were then used to fit a local polynomial form to obtain an optimum prediction of the spectroscopic properties at the correlated ab initio level. Table 


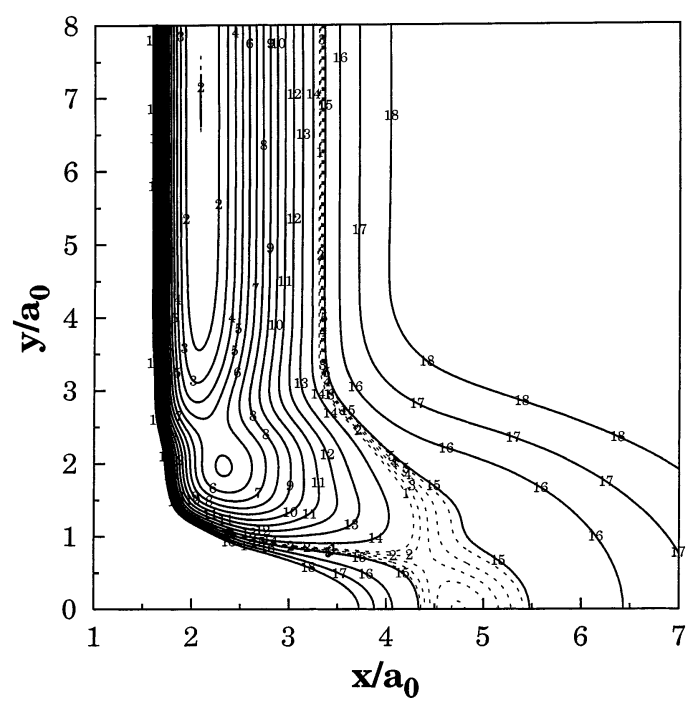

Figure 8. Isoenergy contour plot for the $C_{2 v}$ insertion of a $\mathrm{H}$ atom into a $\mathrm{N}_{2}$ diatomic. Contours are equally spaced by $0.02 E_{\mathrm{h}}$, starting at $-0.3641 E_{\mathrm{h}}$. Contours in dashed lines are equally spaced by $0.002 E_{\mathrm{h}}$, starting at $-0.0932 E_{\mathrm{h}}$.

TABLE 6: Geometries and Energies of Other Important Stationary Points

\begin{tabular}{|c|c|c|c|}
\hline \multirow[b]{2}{*}{ property } & \multirow[b]{2}{*}{$\begin{array}{c}\text { van der Waals, } \\
\mathrm{H} \cdots \mathrm{N}_{2}\end{array}$} & \multicolumn{2}{|c|}{ linear TS } \\
\hline & & $\mathrm{N}-\mathrm{H} \cdots \mathrm{N}$ & $\mathrm{N} \cdots \mathrm{H} \cdots \mathrm{N}$ \\
\hline$R_{1}\left(a_{0}\right)$ & 2.076 & 6.379 & 4.740 \\
\hline$R_{2}\left(a_{0}\right)$ & 7.231 & 1.943 & 2.370 \\
\hline$R_{3}\left(a_{0}\right)$ & 7.231 & 4.436 & 2.370 \\
\hline$V\left(E_{\mathrm{h}}\right)$ & -0.3642 & -0.1338 & -0.0933 \\
\hline$\Delta V(\mathrm{kcal} / \mathrm{mol})$ & $-0.07^{a}$ & $2.26^{b}$ & $23.15^{b}$ \\
\hline$\omega_{1}(\mathrm{~N}-\mathrm{H})\left(\mathrm{cm}^{-1}\right)$ & 55 & 618 & $2163 i$ \\
\hline$\omega_{2}(\mathrm{~N}-\mathrm{N})\left(\mathrm{cm}^{-1}\right)$ & 2335 & 4711 & 538 \\
\hline$\omega_{3}($ bend $)\left(\mathrm{cm}^{-1}\right)$ & 14 & $138 i$ & 978 \\
\hline
\end{tabular}

6 summarizes the properties obtained for other relevant stationary points, including the shallow van der Waals minimum in Figure 5 for intermediate $\mathrm{H}-\mathrm{N}_{2}$ distances.

Clearly, the current $\mathrm{HN}_{2}$ DMBE potential energy surface provides an accurate representation of the properties of the global minimum and the two most important transition states: one that is associated with the reaction $\mathrm{H}+\mathrm{N}_{2} \rightarrow \mathrm{NH}+\mathrm{N}$, and another that is associated with the $\mathrm{HN}_{2}$ isomerization process. Specifically, the barrier height for the process $\mathrm{HN}_{2} \rightarrow$ $\mathrm{H}+\mathrm{N}_{2}$ is predicted to be $10.6 \mathrm{kcal} / \mathrm{mol}$ before inclusion of the zero-point energy correction. Thus, it lies slightly below the $11.3 \mathrm{kcal} / \mathrm{mol}$ extrapolation of Walch and Partridge, ${ }^{10}$ while being very similar to the result reported by $\mathrm{Gu}$ et al. ${ }^{13}$ These authors have used an aug-cc-pVQZ basis set and the CCSD(T) method, having predicted a barrier height of $10.7 \mathrm{kcal} / \mathrm{mol}$. As they also observed, experience suggests that such a barrier may be further reduced when larger basis sets and higher levels of theory are used. ${ }^{8,13}$ The fact that our prediction lies slightly below the best-available theoretical estimates may then provide an indication that the DMBE-SEC method can reliably be used to extrapolate the ab initio energies to the complete basis set/ configuration interaction limit.

We now turn to the global minimum, which is predicted in the present work to be $4.5 \mathrm{kcal} / \mathrm{mol}$ above the $\mathrm{H}+\mathrm{N}_{2}$ asymptote. Thus, it lies close to the Walch and Partridge ${ }^{10}$ extrapolation of $4.31 \mathrm{kcal} / \mathrm{mol}$ and only slightly above the $\mathrm{Gu}$ et al. ${ }^{13}$ prediction of $3.8 \mathrm{kcal} / \mathrm{mol}$. This result may not be surprising, because the literature shows that the estimated values for the barrier height have a tendency to show a more stable trend $(\sim 11 \mathrm{kcal} / \mathrm{mol})$ than the calculated exothermicity for the reaction $\mathrm{HN}_{2} \rightarrow \mathrm{H}+\mathrm{N}_{2}$. We may attribute such a behavior to the fact that the exothermicity is dependent on the level of ab initio theory and basis set used in a more subtle way $3,8,10,13$ (the best reported values cover the range of $3.0-5.6 \mathrm{kcal} / \mathrm{mol}$ ). In fact, the calculated values reflect the dificulties that are usually encountered by the theoretical methods in describing both the short-range and long-range interactions in a balanced way.

The saddle point for the $\mathrm{HN}_{2}$ isomerization process is located at a $\mathrm{T}$-shaped configuration prior to the conical intersection, lying $48.5 \mathrm{kcal} / \mathrm{mol}$ above the $\mathrm{H}+\mathrm{N}_{2}$ asymptote. Both such features are visible in Figure 5, being located along the $y$-axis at $\sim 2.0 a_{0}$. Thus, our estimated barrier height is somewhat smaller than the value of $51.6 \mathrm{kcal} / \mathrm{mol}$ that was reported by Walch, ${ }^{8}$ which may have implications on the lifetime of the $\mathrm{HN}_{2}$ metastable species.

As shown in Figure 5, the present calculations predict a $C_{2 v}$ van der Waals minimum with a well depth of $0.07 \mathrm{kcal} / \mathrm{mol}$, relative to the $\mathrm{H}+\mathrm{N}_{2}$ asymptote. It is a very shallow minimum, which indicates an almost-free rotation of the $\mathrm{H}$ atom around $\mathrm{N}_{2}$. Thus, in reality, the correct location of the lowest energy that is associated with such a weakly bound structure can hardly be warranted to correspond to a $C_{2 v}$-type structure, despite the care taken in performing the least-squares fit and the appreciable number (46 in total) of ab initio points that have been calculated for $r_{\mathrm{H}-\mathrm{N}_{2}} \geq 6.5 a_{0}$.

Tables 5 and 6 show that the spectroscopic properties of all the stationary points in the DMBE potential energy surface are similar to the frequencies obtained when a Taylor-series-type expansion is instead used to fit a dense grid of energies in the vicinity of such points. Indeed, the average percentage error in the calculated frequencies is $1.0 \%$ for the global minimum, saddle point for the $\mathrm{H}+\mathrm{N}_{2}$ reaction, and saddle point for the $\mathrm{HN}_{2}$ isomerization.

Figure 7 shows a contour plot for the linear $\mathrm{N}-\mathrm{H}-\mathrm{N}$ stretching. The main features are the two equivalent $\mathrm{NH} \cdots \mathrm{N}$ hydrogen-bond type structures, and the saddle point for the $\mathrm{H}$-atom exchange process. Note that such structures are not true minima in three-dimensional configuration space, but rather are linear transition states, as indicated by the dashed contours in Figure 6 . Indeed, they have a positive curvature with respect to $\mathrm{NH} \cdot \cdots \mathrm{N}$ stretching but a negative one with respect to bending. Table 6 summarizes the geometries, energies, and vibrational frequencies of such stationary points.

Finally, in Figure 8, we show a countor plot for the $C_{2 v}$ insertion of an $\mathrm{H}$ atom into $\mathrm{N}_{2}$. Although the dashed contours might suggest the resulting linear configuration to be a minimum, it is indeed a saddle point, because it has a negative curvature with respect to the bending mode ( $\mathrm{H}$-atom insertion). In fact, such a "minimum" corresponds, in reality, to the saddle point that connects the two $\mathrm{NH} \cdots \mathrm{N}$ hydrogen-bond type structures that are observed in Figure 7. Also visible from Figure 8 is a shallow "minimum" located at $r_{\mathrm{H}-\mathrm{N}_{2}} \approx 2.0 a_{0}$, which is related to the saddle point that connects the two equivalent metastable structures shown in Figure 5. Finally, for large atomdiatom separations, we observe the very shallow $\mathrm{H} \cdots \mathrm{N}_{2}$ van der Waals minimum that has been already discussed previously (see Figure 5 and Table 6).

\section{Concluding Remarks}

We have reported a single-valued DMBE potential energy surface for ground-state $\mathrm{HN}_{2}$, on the basis of a realistic 
representation of the long-range forces and a fit to accurate ab initio calculations. The various topographical features of the new potential energy surface have been carefully examined and compared with previous calculations for the title system, most importantly, a potential energy surface that was previously reported by Walch and collaborators. $5,6,8,9$ The geometries and spectroscopy of the main stationary points for the $\mathrm{HN}_{2}$ global minimum, transition state for dissociation, and isomerization barrier height are reproduced very accurately when compared with the best available theoretical estimates. We emphasize that the predicted barrier height for dissociation lies below the best available estimates, ${ }^{10,13}$ which suggests that the DMBE-SEC method offers a reliable means to extrapolate accurate correlated energies to the basis set/configuration interaction limit. Thus, we speculate that dynamics calculations that use the new potential energy surface are likely to predict a lifetime for the $\mathrm{HN}_{2}$ species that is even shorter than previously reported estimates. Because the global minimum is predicted to lie 4.5 $\mathrm{kcal} / \mathrm{mol}$ above the $\mathrm{H}+\mathrm{N}_{2}$ asymptote (which is slightly above the best estimates reported in the literature), we further argue that the stability of such a structure is yet unresolved, although it definitely seems to be a metastable one. To summarize, the DMBE potential energy surface reported in the present work is globally valid and accurately fits our ab initio calculations and, for that reason, is being recommended for future dynamics studies of the $\mathrm{H}+\mathrm{N}_{2} \rightarrow \mathrm{HN}_{2} \rightarrow \mathrm{HN}+\mathrm{N}$ reaction.

Acknowledgment. This work has the financial support of European Community, through Contract No. HPRN-CT-200200170, and Fundação para a Ciência e a Tecnologia, Portugal.

\section{References and Notes} 43.

(1) Miller, J. A.; Branch, M. C.; Kee, R. J. Combust. Flame 1981, 81,

(2) Miller, J. A.; Bowman, C. G. Prog. Energy Combust. Sci. 1989, $15,287$.
(3) See ref 5 and references therein.

(4) Glarbor, P. G.; Miller, J. A.; Kee, R. J. Combust. Flame 1986 177,65 .

(5) Walch, S. P.; Duchovic, R. J.; Rohlfing, C. M. J. Chem. Phys. 1989, 90,3230 . 4130 .

(6) Koizumi, H.; Schatz, G. C.; Walch, S. P. J. Chem. Phys. 1991, 95,

(7) Guo, Y.; Thompson, D. L. J. Chem. Phys. 2002, 116, 3670.

(8) Walch, S. P. J. Chem. Phys. 1990, 93, 2384.

(9) Walch, S. P. J. Chem. Phys. 1991, 95, 4277.

(10) Walch, S. P.; Partridge, H. Chem. Phys. Lett. 1995, 233, 331.

(11) Dunning, T. H., Jr. J. Chem. Phys. 1989, 90, 1007.

(12) Kendall, R.; Dunning, T., Jr.; Harrison, R. J. Chem. Phys. 1992, 96,6769 .

(13) Gu, J.; Xie, Y.; Schaefer, H. F., III. J. Chem. Phys. 1998, 108, 8029 .

(14) Varandas, A. J. C. J. Mol. Struct. Theochem. 1985, 120, 401.

(15) Varandas, A. J. C. Adv. Chem. Phys. 1988, 74, 255.

(16) Varandas, A. J. C. Chem. Phys. Lett. 1992, 194, 333

(17) Varandas, A. J. C. Lecture Notes in Chemistry; Laganá, A., Riganelli, A., Eds.; Springer: Berlin, 2000; Vol. 75, p 33.

(18) Varandas, A. J. C. J. Chem. Phys. 1989, 90, 4379.

(19) Werner, H.-J.; Knowles, P. J. J. Chem. Phys. 1988, 89, 5803.

(20) Knowles, P. J.; Werner, H.-J. Chem. Phys. Lett. 1985, 115, 259.

(21) Werner, H.-J.; Knowles, P. J. MOLPRO, 1998. (MOLPRO is a package of $a b$ initio programs written by H.-J. Werner and P. J. Knowles, with contributions from J. Almlöf, R. D. Amos, M. J. O. Deegan, S. T. Elbert, C. Hampel, W. Meyer, K. A. Peterson, R. Pitzer, A. J. Stone, P. R. Taylor, and R. Lindh.)

(22) Varandas, A. J. C.; Silva, J. D. J. Chem. Soc. Faraday Trans. 1992 $88,941$.

(23) Varandas, A. J. C. Mol. Phys. 1987, 60, 527.

(24) Le Roy, R. J. Mol. Spectrosc. (Chem. Soc. London) 1973, 1, 113.

(25) Edwards, S.; Roncin, J.; Launay, F.; Rostas, F. J. Mol. Spectrosc. 1993, $162,257$.

(26) Varandas, A. J. C.; Rodrigues, S. P. J. J. Chem. Phys. 1997, 106, 9647.

(27) Stallcop, J. R.; Bauschlicher, C. W., Jr.; Partridge, H.; Langhoff, S. R.; Levin, E. J. Chem. Phys. 1992, 97, 5578.

(28) Varandas, A. J. C. J. Chem. Phys. 1996, 105, 3524.

(29) Varandas, A. J. C.; Rodrigues, S. P. J. Chem. Phys. Lett. 1995 245,66 .

(30) Matías, M. A.; Varandas, A. J. C. Mol. Phys. 1990, 70, 623.

(31) Martínez-Nuñez, E.; Varandas, A. J. C. J. Phys. Chem. 2001, 105, 